\title{
THE APPLICATION OF REFERENCE-PATH CONTROL TO VEHICLE PLATOONS
}

\author{
Drago Matko, Gregor Klančar, Sašo Blažič \\ Faculty of electrical engineering University of Ljubljana, Slovenia \\ drago.matko@fe.uni-lj.si,gregor.klancar@fe.uni-lj.si, saso.blazic@fe.uni-lj.si \\ Olivier Simonin \\ lab: LORIA Maia project, University of Henri Poincare, Nancy, France \\ olivier.simonin@loria.fr \\ Franck Gechter, Jean-Michel Contet, Pablo Gruer \\ Systems and Transportation Laboratory (SET), University of Technology of Belfort-Montbéliard (UTBM), Belfort, France \\ franck.getcher@utbm.fr, jean-michel.contet@utbm.fr,pablo.gruer@utbm.fr
}

Keywords: Platoon, reactive multiagent, longitudinal and lateral control, reference-path following control.

\begin{abstract}
A new algorithm for the control of vehicle platooning is proposed and tested on a robot-soccer test bed. We considered decentralized platooning, i.e., a virtual train of vehicles, where each vehicle is autonomous and decides on its motion based on its own perceptions. The platooning vehicles have non-holonomic constraints. The following vehicle only has information about its own orientation and about its distance and azimuth to the leading vehicle. Its position is determined using odometry and a compass. The reference position and the orientation of the following vehicle are determined by the estimated path of the leading vehicle in a parametric polynominal form. The parameters of the polynominals are determined using the least-squares method. This parametric reference path is also used to determine the feed-forward part of the applied control algorithm. The feed-back control consists of a state controller with three inputs: the longitudinal and lateral position errors and the orientation error. The results of the experiments demonstrate the applicability of the proposed algorithm for vehicle platoons.
\end{abstract}

\section{INTRODUCTION}

Vehicle platoon systems are a promising approach for new transportation systems because of their innovative capabilities. Their main goals, when applied to passenger cars are (i) an increase in the vehicle density on the highway (i.e., avoiding traffic jams), and (ii) security improvements thanks to automated or semi-automated driving assistance (adaptive cruise control, obstacle detection and avoidance, automatic car parking, etc.). Most of these platooning systems are based on a linear configuration (i.e., a virtual train of vehicles).

Among the several problems associated with the control of platooning systems, longitudinal and lateral control are the most important.

Longitudinal control involves controlling the braking and acceleration in order to stabilize the distance between the leading vehicle and the following vehicle. This control takes as a parameter the distance between the leading and the following ve- hicles. Sheikholeslam and Desoer (Sheikholeslam and Desoer, 1993) proposed a form of longitudinal control based on linearization methods. Ioannou and $\mathrm{Xu}$ (Ioannou and $\mathrm{Xu}, 1994$ ) controlled the brakes and the acceleration using a fixed-gain PID control with gain scheduling. In contrast, Hedrick, Tomizuka and Varaiya (Hedrick et al., 1994) used a control mode based on a non-linear method with PID. Lee, Tomizuka, Jung and Kim (Lee and Tomizuka, 2003; Lee et al., 2000) proposed a longitudinal control based on fuzzy logic.

Lateral control involves aligning the vehicle's direction relative to the vehicle in front. Daviet and Parent (Daviet and M.Parent, 1996) proposed a form of lateral control using a PID controller. This control consists of keeping the angle between the leading and the following vehicles close to zero. In the literature, papers can be found dealing with lateral and longitudinal control using physics-inspired models. For instance, Gehrig and Stein (Gehrig and Stein, 2001) designed a model based on particles' 
submissive forces, whereas $\mathrm{Yi}$ and Chong (Yi and Chong, 2005) developed an impedance-control immaterial hook model. Halle and Chaib-draa (Halle and Chaib-draa, 2005) used a Multi-Agent System (MAS) in order to model immaterial vehicles using constant values from (Daviet and M.Parent, 1996). Contet, Gechter, Gruer and Koukam (Contet et al., 2007) proposed a solution for longitudinal and lateral control using Newtonian forces in an interactive model. In Bom et. all (Bom et al., 2005) a global platooning control strategy is proposed using nonlinear control law which decouples lateral and longitudinal control.

In this paper a novel approach to a platoon of nonholonomic vehicles using the well-known state-space control of nonholonomic systems is presented. The vehicle platooning control strategy relays on relative information to preceding vehicles only therefore no explicit inter-vehicle data exchange and global global information (such as GPS) is required. The important advantage here is that relative information can be measured with low cost sensor sets. Additionally the method to obtain on-line objectives for the follower vehicles control is presented, where the intervehicle distance is curvilinear one as also proposed in (Bom et al., 2005). The proposed control algorithm was tested in simulations and on a platoon of soccer robots.

Controlling nonholonomic systems as they follow a reference path is a well-known problem that has been studied by many authors (Kolmanovsky and McClamroch, 1995; Luca and Oriolo, 1995; Sarkar et al., 1994). The control of vehicles, especially mobile robots, by considering only the first-order kinematics is very common in the literature ((Canudas de Wit and Sordalen, 1992; Oriolo et al., 2002; Balluchi et al., 1996)) as well as in practice. The vehicle has to consider nonholonomic constraints, so its path cannot be arbitrary. Moreover in an environment with obstacles, limitations and other demands the vehicle should be controlled on a reference path, which should follow all the kinematic constraints and avoids obstacles.

The paper is organized as follows: In Section 2 a model of nonholonomic systems and the corresponding control law that can be applied to such systems are presented. The application of the proposed control law to platoon systems is derived in Section 3. The results of the tests on a robot-soccer set-up are presented in Section 4.

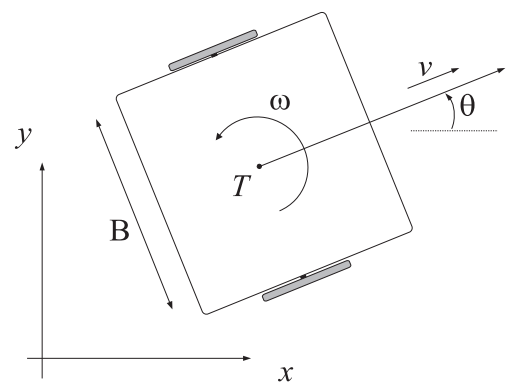

Figure 1: Vehicle architecture and symbols.

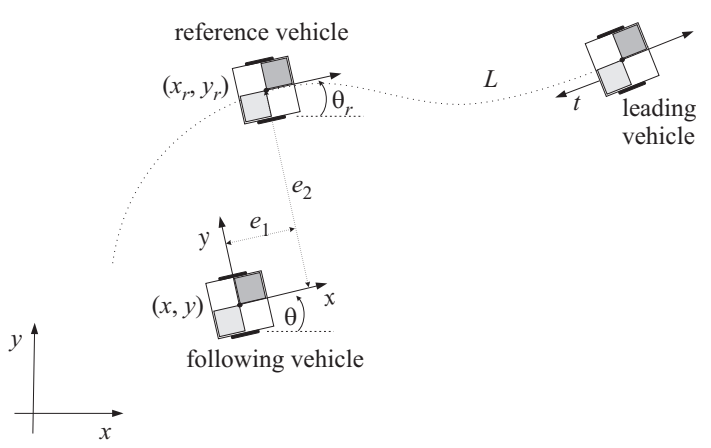

Figure 2: Illustration of the error transformation.

\section{MODELING AND CONTROL OF NONHOLONOMIC SYSTEMS}

In the following the direct and inverse kinematics for mobile vehicles with a differential drive are determined. The vehicle's architecture, together with its symbols, is shown in the Fig. 1, where it is supposed that the geometrical centre $T$ and the centre of gravity coincide.

The equations of motion are as follows

$$
\left[\begin{array}{l}
\dot{x} \\
\dot{y} \\
\dot{\theta}
\end{array}\right]=\left[\begin{array}{ll}
\cos \theta & 0 \\
\sin \theta & 0 \\
0 & 1
\end{array}\right] \cdot\left[\begin{array}{l}
v \\
\omega
\end{array}\right]
$$

where $v$ and $\omega$ are the tangential and angular velocities of the platform shown in the Fig. 1. The right and left velocities of the vehicle's wheels are then expressed as $v_{R}=v+\frac{\omega B}{2}$ and $v_{L}=v-\frac{\omega B}{2}$, respectively, where $B$ is the distance between the robot wheels.

For a given reference trajectory $\left(x_{r}(t), y_{r}(t)\right)$ defined in the time interval $t \in[0, T]$ the feed-forward control law can be derived. From the obtained inverse kinematics the vehicle inputs are calculated, these drive the vehicle on the desired path only if there are no disturbances and no initial state errors. The required vehicle inputs, the tangential velocity $v_{f f}$ and 


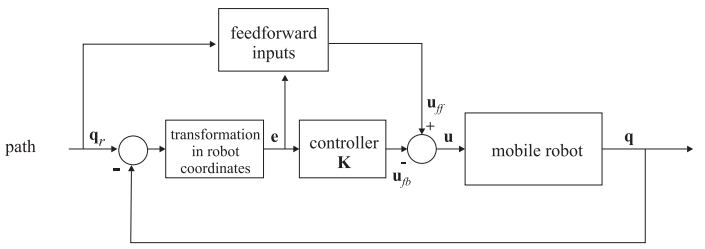

Figure 3: Mobile-vehicle control schematic.

the angular velocity $\omega_{f f}$, are calculated from the reference path. The tangential velocity is given by

$$
\begin{aligned}
v_{f f}(t) & =\sqrt{\dot{x}_{r}^{2}(t)+\dot{y}_{r}^{2}(t)} \\
\omega_{f f}(t) & =\frac{\dot{x}_{r}(t) \ddot{y}_{r}(t)-\dot{y}_{r}(t) \ddot{x}_{r}(t)}{\dot{x}_{r}^{2}(t)+\dot{y}_{r}^{2}(t)}
\end{aligned}
$$

When a vehicle is controlled to drive on a reference path, it usually has some following error. This following error, expressed in terms of the real vehicle, as shown in the Fig. 2, is given by

$$
\left[\begin{array}{l}
e_{1} \\
e_{2} \\
e_{3}
\end{array}\right]=\left[\begin{array}{ccc}
\cos \theta & \sin \theta & 0 \\
-\sin \theta & \cos \theta & 0 \\
0 & 0 & 1
\end{array}\right] \cdot\left[\begin{array}{l}
x_{r}-x \\
y_{r}-y \\
\theta_{r}-\theta
\end{array}\right]
$$

In the Fig. 2 the reference vehicle is an imaginary vehicle that ideally follows the reference path. In contrast, the real vehicle (when compared to the reference vehicle) has some error when following the reference path. Therefore, the control algorithm was designed to force the vehicle to follow the reference path precisely as proposed in (Luca and Oriolo, 1995; Oriolo et al., 2002). It is as follows

$$
\begin{aligned}
& v_{r}=v_{f f} \cos e_{3}-v_{f b} \\
& \omega_{r}=\omega_{f f}-\omega_{f b}
\end{aligned}
$$

where $v_{r}$ and $\omega_{r}$ are reference velocities (set-points) for the low level control controlling the wheels of the vehicle and $v_{f b}, \omega_{f b}$ are the outputs of the feed-back controller given by

$$
\left[\begin{array}{l}
v_{f b} \\
\omega_{f b}
\end{array}\right]=\left[\begin{array}{lll}
-k_{1} & 0 & 0 \\
0 & -\operatorname{sign}\left(u_{f f}\right) k_{2} & -k_{3}
\end{array}\right] \cdot\left[\begin{array}{l}
e_{1} \\
e_{2} \\
e_{3}
\end{array}\right]
$$

The schematic of the obtained control is explained in Fig. 3. The gains $k_{1}, k_{2}$ and $k_{3}$ of the state feedback controller $\mathbf{K}$ were determined by trial and error.

\section{APPLICATION OF THE CONTROLLER TO A LINEAR PLATOON}

It is supposed that there is no data communication between the leading and following vehicles. The following vehicle measures the distance and the azimuth (relative to its own orientation)) of the leading vehicle. To ensure stable control also a measurement of the orientation of the following vehicle (e.g. with a compass) is also needed. No other sensors (e.g., GPS) are required. All the positions are treated in a coordinate system that is fixed to the ground. The following vehicle determines its own position using odometry. Having the current position $\mathbf{X}(k)=[x(k), y(k)]^{T}$, the position in the next sample is determined by a simple Euler integration

$$
\mathbf{X}(k+1)=\mathbf{X}(k)+\left[\begin{array}{c}
\cos (\theta) \\
\sin (\theta)
\end{array}\right] v_{r e f} \Delta t
$$

where $\theta$ is the orientation of the following vehicle, $v_{r e f}$ is the reference speed of the vehicle and $\Delta t$ is the sample time. As shown later, the method of integration and the associated errors in the accuracy of the absolute position are not significant, since only the relative position of both vehicles is important.

The path of the leading vehicle $\mathbf{X}_{h}(k)=$ $\left[x_{h}(k) y_{h}(k)\right]^{T}$ is calculated by the following vehicle using its current position and the measurements of the distance $D$ and the azimuth $\theta_{a}$ (e. g., by using a laser range finder) as follows:

$$
\mathbf{X}_{h}(k)=\mathbf{X}(k)+\left[\begin{array}{c}
\cos \left(\theta+\theta_{a}\right) \\
\sin \left(\theta+\theta_{a}\right)
\end{array}\right] D
$$

This information is stored in the memory and represented in parametric form (with the parameter $k$ - related in the time $t=k \Delta t$ ). The following vehicle is supposed to track the leading vehicle at a distance $L$ - measured on the path of the leading vehicle. First, the time $\mathrm{T}$ needed by the leading vehicle to drive the distance $L$ is calculated using

$$
L=\int_{0}^{T} \sqrt{\dot{x}_{h}^{2}+\dot{y}_{h}^{2}} d t
$$

This time $\mathrm{T}$ is calculated by a linear interpolation of the two successive time instants $(k+1$ and $k)$ defining the time internal where the numerically calculated distance $L^{\prime}$ becomes greater than the desired distance L.

$L^{\prime}=\sum_{k=0}^{N} \sqrt{\left[x_{h}(k+1)-x_{h}(k)\right]^{2}+\left[y_{h}(k+1)-y_{h}(k)\right]^{2}}$ 
According to relation (4) the interpolated value for time $T$ is obtained by $T=k \Delta t+\frac{\Delta t}{L^{\prime}(k+1)-L^{\prime}(k)}(L-$ $\left.L^{\prime}(k)\right)$, where $L$ is the desired tracking distance among the vehicles. Next, the path shape of the leading vehicle at the moment $-T$ ( $T$ seconds in the past) is expressed in the parametric polynomial form

$$
\begin{aligned}
& x_{h}(t)=a_{2}^{x} t^{2}+a_{1}^{x} t+a_{0}^{x} \\
& y_{h}(t)=a_{2}^{y} t^{2}+a_{1}^{y} t+a_{0}^{y}
\end{aligned}
$$

The coefficients of the polynomials $a_{i}^{x}$ and $a_{i}^{y}$ are calculated using the least-squares method with more than three samples around the time $T$ (seven were used in our experiments). The reference position and the orientation of the following vehicle are determined using

$$
\begin{gathered}
\mathbf{X}_{r}=\left[\begin{array}{c}
x_{h}(T) \\
y_{h}(T)
\end{array}\right]=\left[\begin{array}{c}
a_{2}^{x} T^{2}+a_{1}^{x} T+a_{0}^{x} \\
a_{2}^{y} T^{2}+a_{1}^{y} T+a_{0}^{y}
\end{array}\right] \\
\theta_{r}=\operatorname{arctg} \frac{2 a_{2}^{y} T+a_{1}^{y}}{2 a_{2}^{x} T+a_{1}^{x}}
\end{gathered}
$$

respectively. In the Fig. 2 they are denoted as the reference vehicle. The tangential and angular velocities of the reference vehicle (needed for the feed-forward control) are

$$
v_{r}(t)=\sqrt{\left(2 a_{2}^{x} T+a_{1}^{x}\right)^{2}+\left(2 a_{2}^{y} T+a_{1}^{y}\right)^{2}}
$$

and

$$
\omega_{r}(t)=\frac{\left(2 a_{2}^{x} T+a_{1}^{x}\right) \times 2 a_{2}^{y}-\left(2 a_{2}^{y} T+a_{1}^{y}\right) \times 2 a_{2}^{x}}{\left(2 a_{2}^{x} T+a_{1}^{x}\right)^{2}+\left(2 a_{2}^{y} T+a_{1}^{y}\right)^{2}}
$$

respectively. For the feed-back control the error vector is given according to Eq. (4) by

$$
e=\left[\begin{array}{ccc}
\cos \theta & \sin \theta & 0 \\
-\sin \theta & \cos \theta & 0 \\
0 & 0 & 1
\end{array}\right]\left[\begin{array}{c}
\mathbf{X}_{r}-\mathbf{X} \\
\theta_{r}-\theta
\end{array}\right]
$$

\section{RESULTS OF THE EXPERIMENTS}

The proposed algorithm was tested on a robot-soccer set-up (see Fig. 4) consisting of three Middle League MiroSot category robots of size $7.5 \mathrm{~cm}$ cubed, a digital color camera and a personal computer. The color camera mounted above the pitch is a global sensor. The vision part of the programme ((Klančar et al., 2004)) processes the incoming image to identify the positions and orientations of the robots. The first, (leading) robot was driven on a prescribed path. The

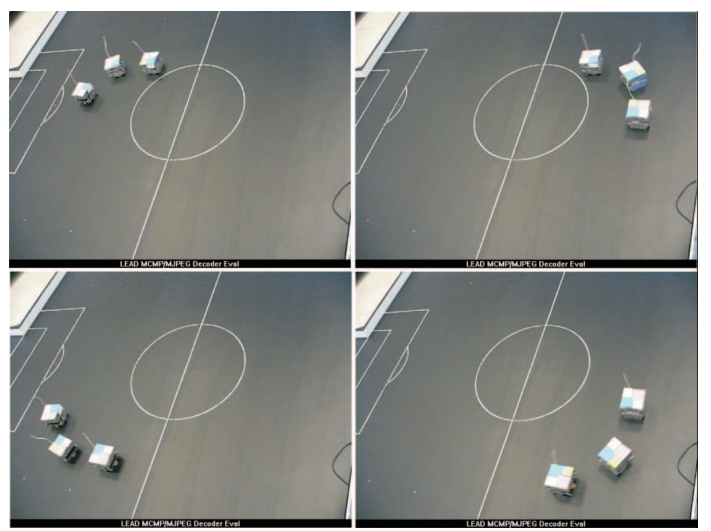

Figure 4: Real set-up experiment.

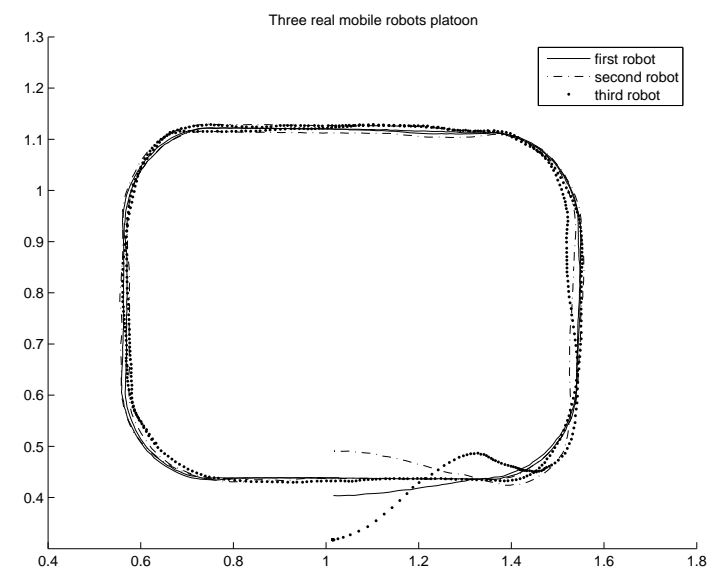

Figure 5: Results of real experiments.

second (the first following) robot receives only the information about its distance and azimuth to the first robot and its own orientation. The third (the second following) robot receives only the information about its distance and azimuth to the second robot and its own orientation. The noisy position estimates of the used camera sensor influences the calculated distance and azimuth information. The estimated noise deviation of measured robots positions was $\pm 5 \mathrm{~mm}$. The distances and azimuth orientations are obtained by $D_{i}=\sqrt{\left(x_{i-1}-x_{i}\right)^{2}+\left(y_{i-1}-y_{i}\right)^{2}}$ and $\theta_{a i}=\arctan \frac{y_{i-1}-y_{i}}{x_{i-1}-x_{i}}$, where $i=2,3$ is robot index.

The parameters values of the controller (5) were $k_{1}=2, k_{2}=20, k_{3}=2$, sampling time was $\Delta t=33 \mathrm{~ms}$ and the desired tracking distance was $L=20 \mathrm{~cm}$. The results of the tests are shown in the Fig. 5. The film of the real experiment can be seen at (Klančar, 2008).

In the Fig. 6 the time course of the distance between the robots is presented. The distance was calculated with assumption that the path between the 


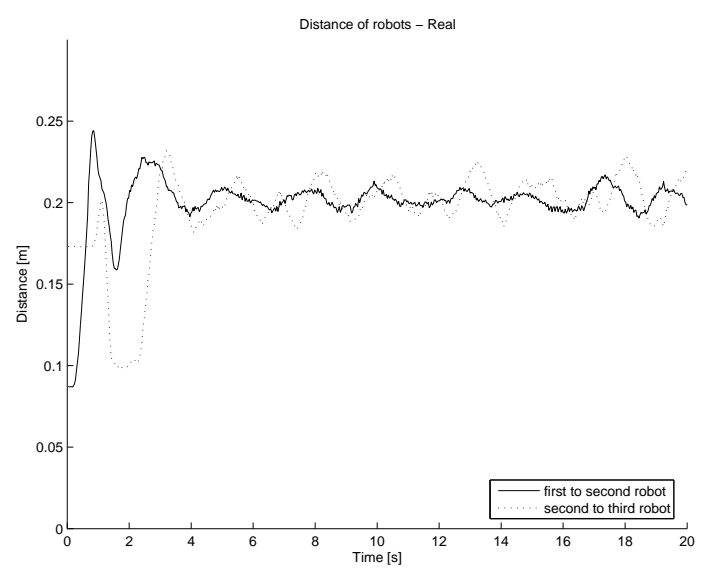

Figure 6: Distance between robots - experiment.

robots is an arc, which results in

$$
L_{\text {arch }}=\frac{\Delta \theta}{2 \sin (\Delta \theta / 2)} \times D
$$

where $\mathrm{D}$ is the straight line between the robots and $\Delta \theta$ is the difference in their orientation angles. It is clear that after a transition phase (the merging and splitting of the platoons is currently under investigation) the second and third vehicle follow with acceptable accuracy. The results of the real experiments are slightly worse due to the noise in the position estimation and due to the time delay of the optical tracking and recognition. The accuracy of the integration method and the associated error, which is equivalent to the slipping of the vehicle's wheels, is analysed and illustrated in the Fig. 7, where the distance between the leading and the following platoon robots in a straight path is illustrated. It can be seen that the constant slipping of the wheels has no influence on the steady-state distance of the platoon vehicles. This conclusion makes sense since servoing accuracy should not be destroyed because relative information among vehicles (distances and azimuth orientations) are always obtained from accurate relative sensor.

\section{CONCLUSIONS}

A new algorithm for the control of vehicle platoons was proposed. The following vehicle only has information about its own orientation and about the distance and azimuth of the leading vehicle. Its own position is determined using odometry and a compass. It calculates the reference path in a parametric polynomial form, and the parameters of the polynomials are determined by the least-squares method. Having the reference path, the feed-forward and feed-back con-

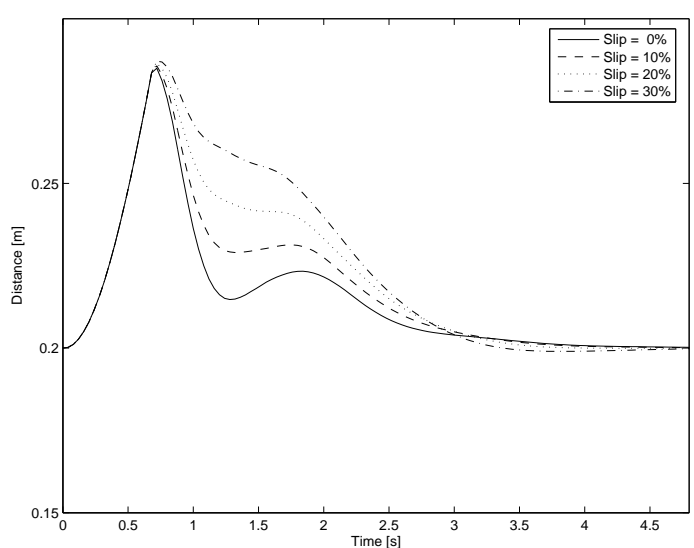

Figure 7: Distance between robots with slip in a straight path (simulation).

trol are applied to the following vehicle. The following vehicle calculates its own position by means of a simple Euler integration. It was established that the error in the integration procedure (equivalent to the errors due the wheel slipping) has a minor influence on the accuracy of the platoon distance. The proposed algorithm was tested on a robot-soccer test bed. The results confirm the applicability of the proposed method.

\section{REFERENCES}

Balluchi, A., Bicchi, A., Balestrino, A., and Casalino, G. (1996). Tracking control for dubin's cars. In Proceedings of the 1996 IEEE International Conference on Robotics and Automation, Minneapolis, Minnesota, pp. 3123-3128.

Bom, J., Thuilot, B., Marmoiton, F., and Martinet, P. (2005). A global control strategy for urban vehicles platooning. relying on nonlinear decoupling laws. In Proceedings of the 2005 IEEE/RSJ International Conference on Intelligent Robots and Systems, Alberta, pp. 1995-2000,.

Canudas de Wit, C. and Sordalen, O. J. (1992). Exponential stabilization of mobile robots with nonholonomic constraints. In IEEE Transactions on Automatic Control, Vol. 37, No. 11, pp. 1791-1797.

Contet, J., Gechter, F., Gruer, P., and Koukam, A. (2007). Application of reactive multiagent system to linear vehicle platoon. In Annual IEEE International Conference on Tools with Artificial Intelligence ( $\left.{ }^{*} I C T A I^{*}\right)$, Grece, Patras.

Daviet, P. and M.Parent (1996). Longitudinal and lateral servoing of vehicles in a pltoon. In IEEE Intelligent Vehicles Symposium, Proceedings, pages 41-46,.

Gehrig, S. K. and Stein, F. (2001). Elastic bands to enhance vehicle following. In IEEE Conference on In- 
telligent Transportation Systems, Proceedings, ITSC, pages 597-602.

Halle, S. and Chaib-draa, B. (2005). A collaborative driving system based on multiagent modeling and simulations. In Transp. Res. C, Emerg. Technol. (UK), 13(4): $320-45$.

Hedrick, J., Tomizuka, M., and Varaiya, P. (1994). Control issues in automated highway systems. In IEEE Control Systems Magazine, 14(6):21-32.

Ioannou, P. and Xu, Z. (1994). Throttle and brake control systems for automatic vehicle following. In IVHS Journal, 1(4):345.

Klančar, G. (2008). http://msc.fe.uni-lj.si/publicwww /klancar/robotsplatoon.html.

Klančar, G., Kristan, M., and S. Kovačič, O. O. (2004). Robust and efficient vision system for group of cooperating mobile robots with application to soccer robots. In ISA Transactions, vol. 43, pp. 329-342.

Kolmanovsky, I. and McClamroch, N. H. (1995). Developments in nonholonomic control problems. In IEEE Control Systems, Vol. 15, No. 6, pp. 20-36.

Lee, H. and Tomizuka, M. (2003). Adaptive vehicle traction force control for intelligent vehicle highway systems (ivhss). In IEEE Transactions on Industrial Electronics, 50(1):37-47.

Lee, M., Jung, M., and Kim, J. (2000). Evolutionary programming-based fuzzy logic path planner and follower for mobile robots. In Proceedings of the IEEE Conference on Evolutionary Computation, ICEC, (1) pp 139-144.

Luca, A. and Oriolo, G. (1995). Modelling and control of nonholonomic mechanical systems. In Kinematics and Dynamics of Multi-Body Systems,SpringerVerlag, Wien.

Oriolo, G., Luca, A., and Vandittelli, M. (2002). Wmr control via dynamic feed-back linearization: Design, implementation, and experimental validation. In IEEE Transactions on Control Systems Technology, Vol. 10, No. 6, pp. 835-852.

Sarkar, N., Yun, X., and Kumar, V. (1994). Control of mechanical systems with rolling constraints: Application to dynamic control of mobile robot. In The International Journal of Robotic Research, Vol. 13, No. 1, pp. 55-69.

Sheikholeslam, S. and Desoer, C. (1993). Longitudinal control of a platoon of vehicles with no communication of lead vehicle information: A system level study. In IEEE Transactions on Vehicular Technology, 42(4):546-554.

Yi, S.-Y. and Chong, K.-T. (2005). Impedance control for a vehicle platoon system. In Mechatronics (UK), 15(5):627-38. 